\title{
Can we use mesenchymal stem cell transplantation for COVID-19 patients in puerperium period?
}

To the Editor,

The Coronavirus disease-2019 (COVID-19) pandemic has affected the whole world, and pregnant women and women in the postpartum period have not been spared from its effects. Pregnant women during this pandemic pose an unknown risk to their babies. We followed up pregnant and post-pregnancy COVID-19 patients in this period in the intensive care unit (ICU) and used mesenchymal stem cell (MSCs) transplantation in suitable patients. We aimed to investigate patients with COVID-19 pneumonia and who were hospitalized in the ICU after C-section in the puerperium period and either given or not given MSCs treatment.

Case 1: A 33 year-old multiparous woman with a history of pregnancy at 33 gestation weeks, admitted with fever ( $>38$ ${ }^{\circ} \mathrm{C}$ ) and cough, with a presumptive diagnosis of COVID-19. Chest computed tomography showed patchy ground-glass opacity (Figure 1). On the third day of hospitalization, she had severe dyspnea and tachypnea and delivery was advised, so she underwent C-section with spinal anesthesia. The patient was transferred to ICU after labor with respiratory distress and X-rays showed patchy ground-glass opacities. She was intubated. D-dimer, C-reactive-protein (CRP), and ferritin levels continued to increase up to the tenth day after the C-section. The patient's clinical situation was evaluated as cytokine storm. After tocilizumab treatment, MSC treatment was given twice with two days intervals. At the end of the third week the patient was extubated. X-rays showed dramatic healing of the patchy ground-glass opacity. When she could breath air unassisted, she was discharged from ICU and subsequently to home.

Case 2: A 34 year-old woman was admitted to ICU after cesarean section with a diagnosis of COVID-19. She had tachypnea and shortness of breath in the postoperative period. High flow nasal cannula oxygenation, intermittent non-invasive ventilation and intermittent prone positioning was applied at
ICU follow up. The patient did not respond to seven days of this tretment so she was intubated. Again, D-dimer, CRP, and ferritin levels continued to increase. On the eleventh and twelfth days, she received immune plasma therapy. After 21 days, despite all these interventions, she unfortunately died.

MSCs are tolerated by the recipient immune system while also being immunomodulatory. MSCs are known to function via several mechanisms relevant to acute lung injury. When administered intravenously they sequester in the lung. The effects of MSC are known to include: anti-inflammatory mechanisms; inhibition of lung fibrosis; lung tissue regeneration; and an anti-apoptotic effect in injured cells (1). After MSC injection, cytokine-secreting immune cells, including NK cells, were markedly reduced and pro-inflammatory cytokine tumor

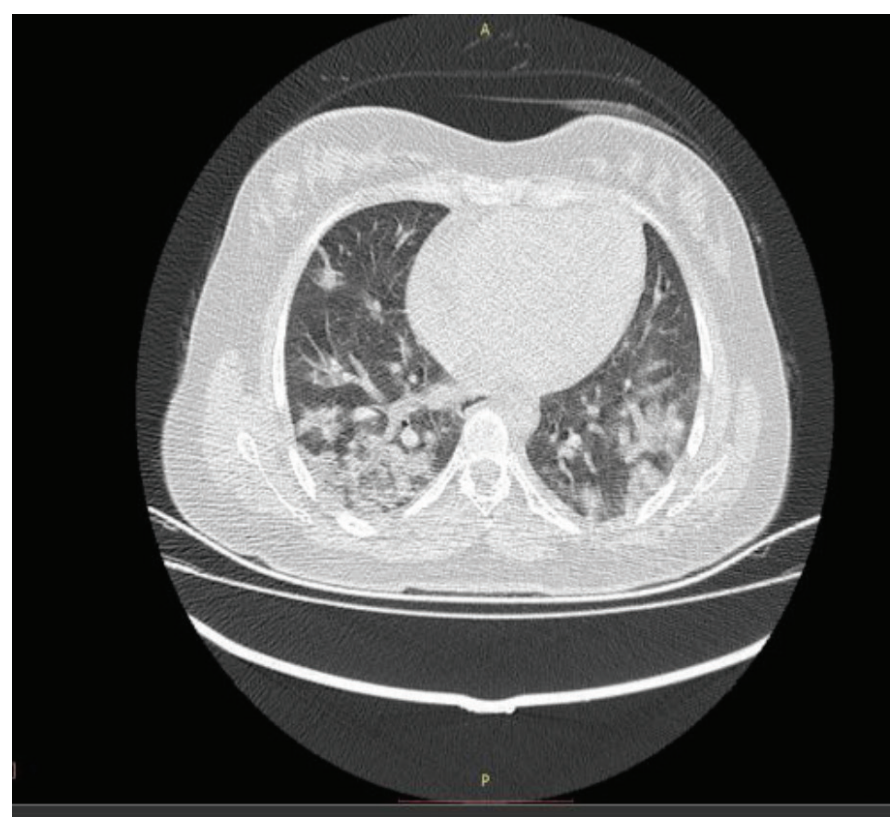

Figure 1. Thorax CT image before ICU admission CT: Computed tomography, ICU: Intensive care unit 
necrosis factor-alpha was decreased (2). MSC transplantation may be an appropriate choice of therapy in patients with severe COVID-19 to prevent severe morbidity and mortality in critically ill patients in the future.

\section{(D) Ayça Sultan Şahin1, (D) Ebru Kaya1, (D) Gürsel Turgut², (D) Ali Kocataş3}

1Department of Anesthesiology and Reanimation, University of Health Sciences Turkey, Kanuni Sultan Süleyman Training and Research Hospital, Istanbul, Turkey

2Department of Plastic and Reconstructive Surgery, Genkord-Umbilical Cord Blood Banking, İstanbul, Turkey
3Department of General Surgery, University of Health Sciences Turkey, Kanuni Sultan Süleyman Training and Research Hospital, İstanbul, Turkey

\section{References}

1. Rogers CJ, Harman RJ, Bunnel BA, Schreiber MA, Xiang C, Wang FS, et al. Rationale for the clinical use of adipose-derived mesenchymal stem cells for COVID-19 patients. J Transl Med 2020; 18: 203.

2. Xiao K, Hou F, Huang X, Li B, Qian ZR, Xie L. Mesenchymal stem cells: current clinical progress in ARDS and COVID-19. Stem Cell Res Ther 2020; 11: 305 